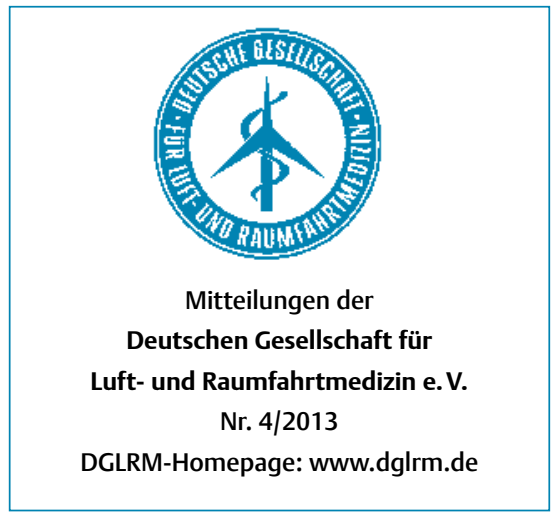

Geschäftsstelle der DGLRM

Frau Doris Mittelstädt - DLR

Linder Höhe

51170 Köln

E-Mail: geschaeftsstelle@dglrm.de

Sprechzeiten: mittwochs 14:00-16:00 Uhr Tel.: 0176/56242876

\title{
Liebe DGLRM-Mitglieder, sehr geehrte Damen und Herren,
}

nun ist es bald so weit, die Jahrestagung der DGLRM im Rahmen der Drei-LänderTagung für Luft- und Raumfahrtmedizin steht vor der Tür. In diesem Jahr stehen unter anderem Vorstandsneuwahlen in der Mitgliederversammlung an. Neben einem interessanten Vortragsprogramm werden auch Round-Table-Diskussionen mit den Vertretern der Luftfahrtbehörden aus Deutschland, Österreich und der Schweiz stattfinden. Das Rahmenprogramm mit Empfang und Besichtigung des Zeppelinhangars sowie der Festabend im Dorniermuseum runden die Tagung ab. Es ist mir eine ganz besondere Freude, dass die Vollversammlung der European Society of Aerospace Medicine am Morgen des 15. September $2013 \mathrm{im}$ Anschluss an die Drei-Länder-Tagung auch im GrafZeppelin-Haus in Friedrichshafen stattfinden wird. Hier bietet sich die Gelegenheit, die Vertreter der ESAM und ihre Arbeit näher kennenzulernen. Ich freue mich auf Ihr Kommen.

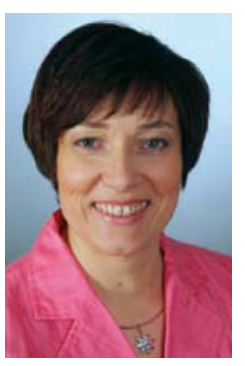

Ihre

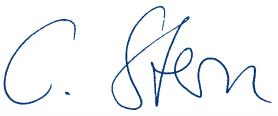

Claudia Stern, Köln

\section{Einladung}

\section{DGLRM-Mitglieder-}

\section{versammlung}

am 13.09.2013 um 15:45 Uhr

im Graf-Zeppelin-Haus

in Friedrichshafen

TOP 1 Eröffnung und Begrüßung

TOP 2 Genehmigung der Tagesordnung

TOP 3 Wahl des Schriftführers

TOP 4 Bericht der Präsidentin

TOP 5 Bericht des Sekretärs

TOP 6 Bericht der Schatzmeisterin

TOP 7 Bericht der Rechnungsprüfer

TOP 8 Entlastung des Vorstands

TOP 9 Beschlussfassung über die Neufassung der Satzung (Version 27.06.2013)

TOP 10 Wahl des Wahlleiters

TOP 11 Neuwahlen des Vorstands

TOP 12 Neuwahlen des Vorstandsrats

TOP 13 Berichte aus den Arbeitsgruppen

TOP 14 Zeit und Ort nächste Jahrestagung

TOP 15 Verschiedenes

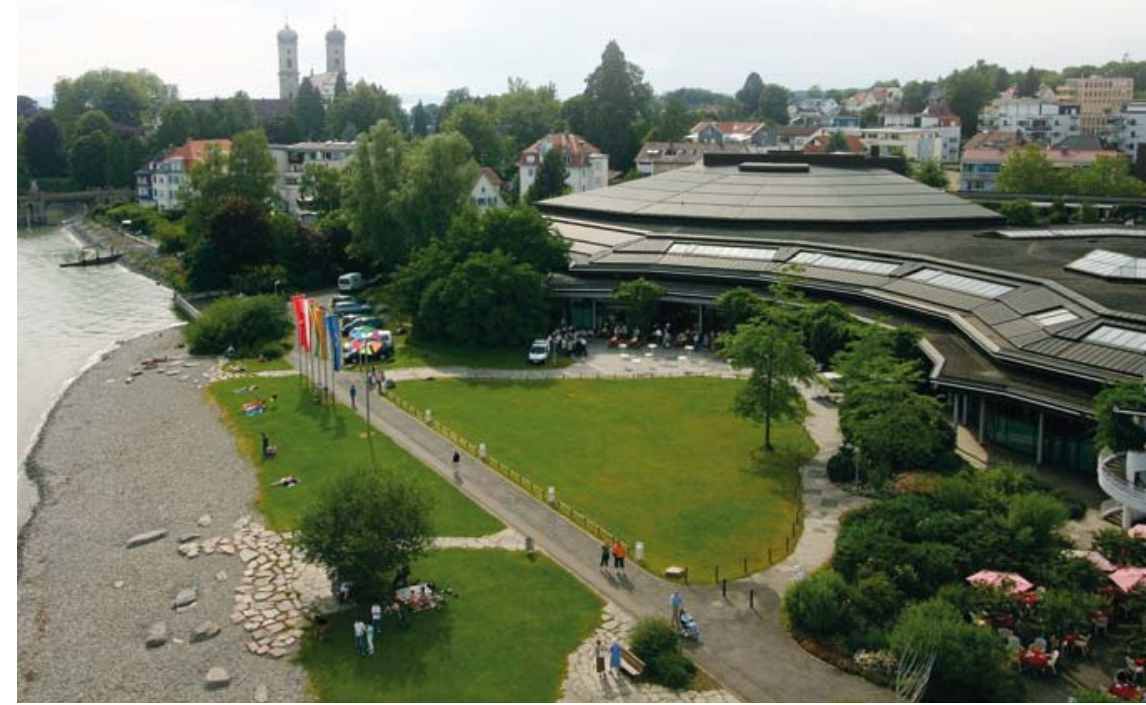

Graf-Zeppelin-Haus, Friedrichshafen 


\section{Won Chuel Kay Award für Professor Dr. Hans Pongratz}

Professor Dr. Hans Pongratz (Mitte) erhielt beim diesjährigen AsMAKongress den Won Chuel Kay Award der koreanischen Luftwaffe für auBerordentliche Verdienste im Bereich der internationalen Flugmedizin.

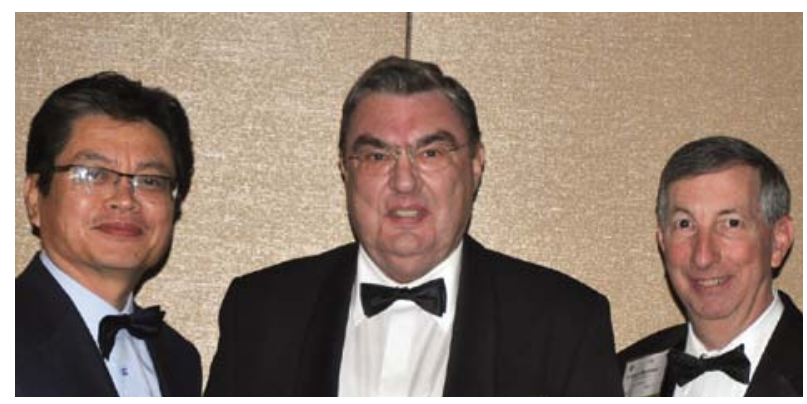

Auf dem diesjährigen 84. Kongress der Aerospace Medical Association (AsMA) in Chicago wurde Honorarprofessor Dipl.Ing. (univ.) Dr. med. Hans Pongratz während des Honors Night Banquet am 16. Mai 2013 unter großer Anteilname der Won Chuel Kay Award verliehen.

Von der koreanischen Luftwaffe zu Ehren von Dr. Won Chuel Kay, dem früheren Generalarzt der koreanischen Luftwaffe, ins Leben gerufen, wird der Award an würdige Preisträger verliehen, die sich außerordentliche Verdienste im Bereich der internationalen Flugmedizin erworben haben.

In der Laudatio wird unter anderem gewürdigt, dass Hans Pongratz, langjähriger Fellow und früherer Vizepräsident der AsMA, lange Jahre erfolgreich im Bereich und der Gestaltung wissenschaftlicher Themen für den Kongress und für internationale Zusammenarbeit tätig war. Nicht zuletzt rief er das deutschsprachige Panel wieder ins Leben, das die Reputation deutschsprachiger Flugmedizin aufgrund hohen wissenschaftlichen Niveaus international festigte.
Hans Pongratz ist Verbindungsoffizier zwischen der europäischen ESAM und der AsMA. In dieser Funktion setzt er sich seit Jahren unermüdlich und sehr erfolgreich für nationale wie internationale wissenschaftliche Zusammenarbeit auf dem Gebiet der Luft- und Raumfahrtmedizin ein. Dabei versteht er es vorzüglich, die Interessen militärischer und ziviler Intentionen $\mathrm{zu}$ bündeln und $\mathrm{zu}$ gemeinsamen Erfolgen zu führen.

Auch als wegweisender Präsident unserer Gesellschaft war es ihm immer ein Anliegen, auf der Grundlage wissenschaftlicher und akademischer Kompetenz eine eigenständige und anerkannte deutsche Flugmedizin zu etablieren. Als Vordenker und Vorreiter erkannte er sehr früh, dass mehr denn je eine erfolgreiche Flugmedizin multidisziplinär und international ausgerichtet sein muss.

Dies wurde jetzt international gewürdigt und honoriert.

Wir gratulieren Hans Pongratz zu dieser herausragenden Ehrung sehr herzlich. Dr. Erich Rödig, Generalarzt a.D.

\section{Kongresse 2013}

Drei-Länder-Tagung für Luft- und Raumfahrtmedizin

Dt. Ges. f. Luft- und Raumfahrtmedizin, Österr. Ges. f. Luftfahrtmedizin,

Swiss Soc. of Aviation Medicine und Mitgliederversammlung der ESAM am 15.09.2013

Termin: 12.09.-15.09.2013

Ort: $\quad$ Friedrichshafen

E-Mail: 3LaenderTagung2013@ m4-cp.de

Internet: www.dglrm.de $61^{\text {st }}$ International Congress of Aviation and Space Medicine (ICASM)

Int. Academy of Aviation and Space Medicine

Termin: 06.10.-10.10.2013

Ort: Jerusalem, Israel

Internet: www.icasm2013.org 


\section{0 teilnehmende Astronauten beim ASE-Kongress in Köln}

Vom 1. bis 5. Juli 2013 fand in Köln der 26. Kongress der Association of Space Explorers statt. 80 Astronauten, Kosmonauten und Taikonauten kamen auf Ein-

ladung des deutschen Astronauten Dr. Reinhold Ewald und dem Leiter des Instituts für Luft- und Raumfahrtmedizin Prof. Rupert Gerzer nach Köln.

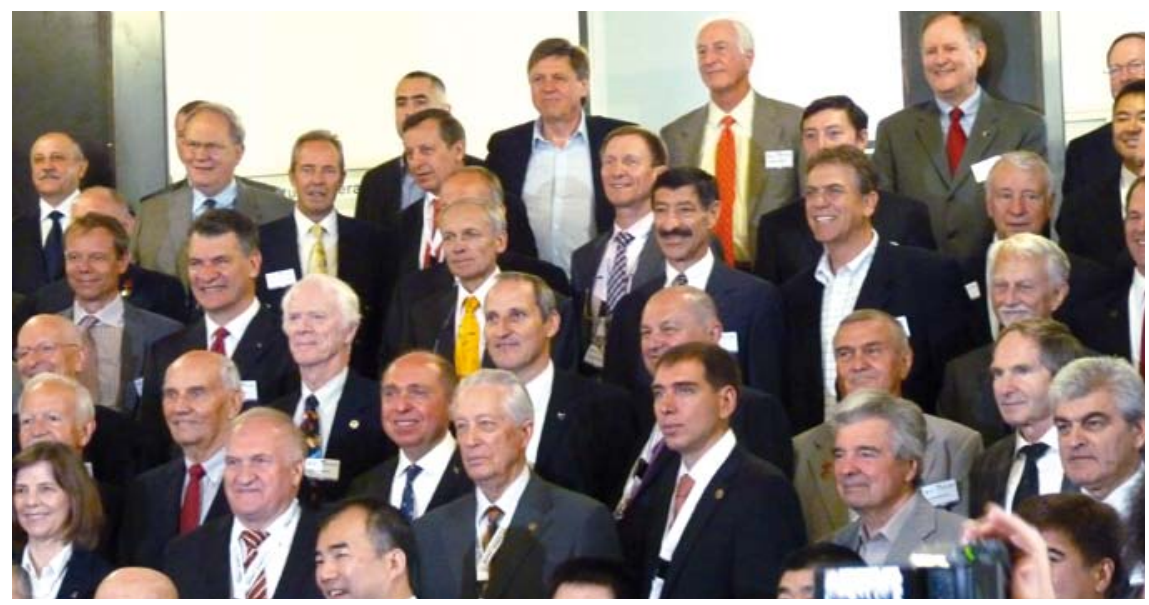

Einige der 80 Astronauten mit 4 europäischen und 2 deutschen Astronauten - haben Sie sie entdeckt?

Fortbildungen 2013

EUSAM - Basic course 23 (englisch)

European School of Aviation Medicine

Termin: 31.08.-08.09.2013

Ort: Frankfurt Airport, Lufthansa-Basis

Kontakt: Sigrid Froese, Tel.: +49 (0)69/69691222

Internet: www.flugmed.org / www.eusam.org

EUSAM - Basic course 23/2 (englisch) European School of Aviation Medicine

Termin: 28.09.-06.10.2013

Ort: Frankfurt Airport, Lufthansa-Basis

Kontakt: Sigrid Froese, Tel.: +49 (0)69/69691222

Internet: www.flugmed.org / www.eusam.org

DAF-Refresher-Seminar Flugmedizin (deutsch)

Dt. Akademie für Flug- und Reisemedizin Termin: 25.10.-27.10.2013

Ort: Seeheim-Jugendheim, Lufthansa Training \& Conference Center
Kontakt: Sigrid Froese, Tel.: +49 (0)6969691222

Internet: www.flugmed.org

EUSAM - Advanced course 23 (englisch)

European School of Aviation Medicine

Termin: 07.12.-15.12.2013 und 08.03.-16.032014

Ort: Frankfurt Airport, Lufthansa-Basis

Kontakt: Sigrid Froese, Tel.: +49 (0)69/69691222

Internet: www.flugmed.org / www.eusam.org

EUSAM - Advanced course 23/2 (englisch)

European School of Aviation Medicine Termin: 08.03.-16.03.2014

Ort: Frankfurt Airport, Lufthansa-Basis

Kontakt: Sigrid Froese, Tel.: +49 (0)69/69691222

Internet: www.flugmed.org / www.eusam.org
Der Kongress wurde am 1. Juli in der Universität Köln eröffnet. Im Rahmen der Eröffnung erhielt Prof. Riesenhuber den Crystal Helmet Award für seine Verdienste um die Unterstützung der Raumfahrt verliehen.

Am Community Day, 3. Juli, schwärmten die Astronauten in 40 unterschiedliche Orte in Deutschland aus, um den Geist der Raumfahrt möglichst weit zu streuen. Sie besuchten sowohl die Bundeskanzlerin als auch Schulen, Universitäten und Forschungseinrichtungen.

Am 5. Juli stand ein Besuch des European Astronaut Centers auf dem Gelände des DLR und die feierliche Eröffnung der neuen Forschungseinrichtung :envihab auf dem Programm. Hierzu waren seit 1990 erstmals wieder alle deutschen Astronauten auf einer Bühne vereint.

\section{Verantwortlich für den Inhalt der DGLRM-Seiten}

Deutsche Gesellschaft für Luft- und

Raumfahrtmedizin e. V. (DGLRM)

Dr. Claudia Stern, Präsidentin, DLR,

51170 Köln, claudia.stern@dlr.de

Redaktionsschluss: 24.07.2013

Bankverbindung

Deutsche Bank Hamburg,

Konto-Nr.: 3889482, BLZ: 20070000

IBAN: DE 63200700240388948200 ,

SWIFT/BIC: DEUTDEDBHAM

Änderungen für die Mitgliederkartei bitte an: Dr. Martin Trammer, DLR,

51170 Köln, martin.trammer@dlr.de

\section{Wir gratulieren}

65 Jahre, Dr. Friedrich Simon, Marienheide, 26.06.1948

70 Jahre, Dr. Wolfram Blank, Lärz, 02.08.1943

70 Jahre, Dr. Eberhard Hennig, Tamm, 27.07.1943

\section{Wir begrüßen als neue Mitglieder}

Swantje Hauschild, Sisseln/Schweiz

Bita Rezazadeh, Aachen

Swantje Tauber, Zürich/Schweiz 